\title{
Vorwort zur 1. Auflage
}

Dieses Lehrbuch gibt eine moderne Darstellung des Stoffs, der typischerweise Gegenstand der Vorlesung Quantenmechanik II ist. Schwerpunkte des Buchs sind neben den Symmetrien, der Streutheorie und der relativistischen Quantenmechanik vor allem die Vielteilchentheorie einschließlich der Quantenstatistik. Ausführlich wird die Zweite Quantisierung behandelt. Die statistischen Ensembles werden aus dem Prinzip der maximalen Entropie abgeleitet. Aufgenommen wurde auch die Berry-Phase, in deren Kontext der Bohm-Aharonov-Effekt erklärt wird. Wie schon in Band 1 wird neben dem traditionellen Operatorformalismus auch die Pfad- oder Funktionalintegralbeschreibung entwickelt, die bisher noch recht wenig Einzug in die Standardkursvorlesungen gefunden hat, aber eine Reihe von konzeptionellen und praktischen Vorzügen besitzt. Detailliert wird deshalb die Funktionalintegralbeschreibung von Bose- und FermiSystemen ausgearbeitet, die den unmittelbaren Einstieg in die Quantenfeldtheorie ermöglicht.

Große Sorgfalt wird auf Verständlichkeit gelegt: Bei den mathematischen Ableitungen werden sämtliche erforderlichen Teilschritte angegeben, sodass das Buch auch zum Selbststudium geeignet ist. Wesentliche Formeln sind besonders markiert, Nebenrechnungen sowie erläuternde Bemerkungen sind grau hinterlegt. Erstmals benutzte Fachbegriffe sowie wichtige Textpassagen sind kursiv gedruckt. Einige Kapitel bzw. Unterkapitel, die für ein Verständnis des restlichen Stoffs nicht unmittelbar notwendig sind und deshalb bei einer ersten Lektüre übergangen werden können, sind mit einem Stern * gekennzeichnet. Zur einfacheren Referenzierung der Kapitel wurden diese fortlaufend von Band 1 nummeriert.

Wie schon im Band 1 wird in diesem Buch durchgängig das in der Quantenfeldtheorie übliche Heaviside-Lorentz-Maßsystem benutzt, das eine Reihe von Vorteilen bietet. In Abweichung von den Gepflogenheiten der Quantenfeldtheorie wird aus didaktischen Gründen jedoch $\hbar$ und $c$ nicht auf Eins gesetzt.

Das vorliegende Buch ist aus Skripten zu Vorlesungen entstanden, die der $\mathrm{Au}$ tor an der TU Dresden und vor allem an der Universität Tübingen gehalten hat. Allen Studenten, die durch ihre konstruktive Kritik zur Verbesserung des Buchs beigetragen haben, sei an dieser Stelle gedankt, auch wenn sie nicht alle namentlich erwähnt werden können. Das gesamte Manuskript wurde von Herrn Marco Herbst aus der Sicht eines Studenten hinsichtlich Verständlichkeit gelesen. Neben zahlreichen Hinweisen zum Inhalt hat er sehr zur Vereinheitlichung der Notation beigetragen. Einige Abbildungen wurden von Dr. Davide Campagnari und Priv. Doz. Dr. Markus Quandt angefertigt. Das LaTeX-Manuskript inklusive Abbildungen wurde von meiner Sekretärin, Frau Ingrid Estiry, erstellt. Ihnen allen sei für ihre mühevolle Arbeit und ihr Engagement gedankt. Mein besonderer Dank gilt Herrn Priv. Doz. Dr. Markus Quandt und Herrn Dr. Davide Campagnari, die das gesamte Manuskript gelesen haben und durch zahl- 
reiche wertvolle Hinweise und Kommentare zur Verbesserung des Buchs beigetragen haben. Schließlich sei dem Verlag für die aufgebrachte Geduld und die angenehme Zusammenarbeit gedankt.

Tübingen, im August 2013 Hugo Reinhardt 